\title{
Frequency of Bacterial Organisms Isolated from Cerebrospinal Fluid (CSF) of Children under Five Years in Windhoek from 2010 to 2014
}

\author{
Erastus Lafimana Haimbodi', Munyaradzi Mukesi ${ }^{*}$, Owen Mtambo², \\ Sylvester Rodgers Moyo ${ }^{1}$ \\ ${ }^{1}$ Department of Health Sciences, Faculty of Health and Applied Sciences, Namibia University of Science and \\ Technology, Windhoek, Namibia \\ ${ }^{2}$ Department of Mathematics and Statistics, Faculty of Health and Applied Sciences, Namibia University of \\ Science and Technology, Windhoek, Namibia \\ Email: *mmukesi@nust.na
}

Received 24 May 2016; accepted 5 September 2016; published 8 September 2016

Copyright (C) 2016 by authors and Scientific Research Publishing Inc.

This work is licensed under the Creative Commons Attribution International License (CC BY). http://creativecommons.org/licenses/by/4.0/

(c) (i) Open Access

\section{Abstract}

Bacterial meningitis (BM) is a life-threatening condition which affects the central nervous system (CNS). Its incidence rate is estimated between $0.22-2.66$ and $0.81-6.1$ cases/1000 lives worldwide and in Africa respectively. The objective of this study was to determine the frequency of bacterial organisms isolated from CSF of children less than five years old in Windhoek. A retrospective analysis was performed on $\mathbf{7 8 4}$ results of CSF submitted to the Namibia Institute of Pathology (NIP) from January 2010 to August 2014. The results showed that out of the suspected meningitis cases, $18(28.6 \%)$ were due to Streptococcus pneumoniae, making it the frequently isolated organism, followed by Staphylococcus aureus 7 (11.1\%), Escherichia coli 5 (7.9\%) and Haemophilus influenzae 4 (6.3\%). Streptococcus pneumoniae showed high resistance to penicillin 17 (70.5\%) \& cotrimoxazole $16(93.7 \%)$ and $100 \%$ susceptibility to ciprofloxacin (5), vancomycin (13) \& ceftriaxone (8). Haemophilus influenzae showed moderate resistance to cotrimoxazole $3(66 \%) \&$ tetracycline $2(50 \%)$. It showed $100 \%$ sensitivity to chloramphenicol (4), cefuroxime (2) \& ceftriaxone (3). Neisseria meningitidis showed high resistance to cotrimoxazole by $100 \%(\mathrm{n}=2)$ and high sensitivity to chloramphenicol $(n=2)$, ceftriaxone \& penicillin by $100 \%(n=2)$. Streptococcus agalactiae was resistant to tetracycline and sensitive to penicillin \& erythromycin by $100 \%$ (n = 1). Streptococcus pneumoniae was isolated the most in this current study and it had high resistance to penicillin \& cotrimoxazole. There was a significant difference between results CSF culture and

${ }^{*}$ Corresponding author.

How to cite this paper: Haimbodi, E.L., Mukesi, M., Mtambo, O. and Moyo, S.R. (2016) Frequency of Bacterial Organisms Isolated from Cerebrospinal Fluid (CSF) of Children under Five Years in Windhoek from 2010 to 2014. Open Journal of Medical Microbiology, 6, 125-132. http://dx.doi.org/10.4236/ojmm.2016.63017 
PCR, Gram stain, CSF cell count, protein $\&$ glucose, as all comparisons yielded in $P$ values less than 0.05 , indicating a significant statistical association.

\title{
Keywords
}

\author{
Bacterial Meningitis, Frequency, Cerebrospinal Fluid, Retrospective, Namibia
}

\section{Introduction}

Meningitis is a result of the intrusion of the meninges by microbial agents. BM claims many lives annually and it is associated with a high incidence of disability and long-term health implications. Furthermore, about $5 \%$ mortality rates have been reported in children from developed countries, whereas in developing countries, the rates have been estimated at $30 \%$ [1]. About $10 \%-20 \%$ of those that survive from BM experience permanent sequelae such as epilepsy, mental retardation and sensorineural deafness [2].

The development of BM usually follows colonization of the respiratory mucosa by invasive bacteria. This mechanism involves attachment of the bacterial organism onto the nasopharyngeal mucosal cells, colonization of the respiratory mucosa, invasion of the intravascular space, bacteraemia, meningeal invasion, bacterial replication in the subarachnoid space, thus leading to inflammation of the subarachnoid space [2] [3]. Newborns, individuals living in low income countries as well as those infected with Gram-negative bacilli and S. pneumoniae are at greater risk of BM [4].

The main bacterial causes of meningitis in neonates (premature and full term) up to 3 months of age include $S$. agalactiae or group B streptococcus (GBS), coliform bacilli such as E. coli possessing K1 antigens, as well as L. monocytogenes [5]. In infants and small children, S. pneumoniae, $N$. meningitidis and H. influenzae type B are predominant meningeal pathogens [5]. S. pneumoniae, $N$. meningitidis and $H$. influenzae type B account for $90 \%$ of reported cases of acute bacterial meningitis in infants and children above 4 weeks of age [2].

Clinical manifestations of bacterial meningitis include fever, headache, hypothermia, nausea, vomiting, photophobia, confusion, lethargy, irritability, poor feeding, respiratory distress, diarrhoea, bulging fontanelles and seizures [4]. Laboratory confirmation of meningitis requires collection of CSF through lumbar puncture, before administration of empirical therapy [2]. Culture, Gram stain, microscopy, macroscopy, cell count, protein \& glucose estimation and polymerase chain reaction (PCR) are some of the methods used for detection of causative agents [6].

In Namibia in 2003, nine cases of BM were reported in neonates less than 1 month, 93 cases in infants aged 1 11 months \& 80 cases in children aged 1 - 5 years. It was reported that Extended Spectrum Beta Lactamase (ESBL) Klebsiella pneumonia was most commonly isolated from CSF collected from neonates. Streptococci, $H$. influenzae and Staphylococci were commonly isolated from CSF collected from infants aged 1 - 11 months. $N$. meningitidis, $H$. influenzae and $S$. pneumoniae were mostly isolated from children aged 1 - 5 years [7].

The study was aimed at investigating the frequency of bacterial organisms isolated from children less than 5 years in Windhoek for the period 2010 to 2014, as well as their antimicrobial susceptibility patterns.

\section{Methodology}

\section{Study Design}

This research was a descriptive retrospective study, conducted to establish the frequency of bacterial organisms isolated from CSF samples of children under the age of five years in Windhoek, Namibia from the year 2010 to 2014. The study included 784 CSF results of children under the age of five years whose CSF samples were examined at the Namibia Institute of Pathology central laboratory in Windhoek, Namibia, from 2010 to 2014. These were children admitted at the Katutura intermediate hospital and Windhoek central hospital. The Katutura intermediate hospital is the referral centre for all hospitals across Namibia and has a high admission rate due to referred cases. The Windhoek central hospital is classified as the national referral hospital handling specialised cases referred from the Katutura hospital and from the whole of Namibia.

All bacterial culture positive and negative results were included in the study. CSF results which were positive 
for fungal, parasitic or viral pathogens were excluded from the study. Follow-up results and results that queried contamination were also excluded from the study. This study was carried out in Windhoek, Namibia.

\section{Materials and Methods}

\subsection{Examination of CSF}

CSF samples were examined at NIP microbiology laboratory using a procedure cited in Cheesbrough, 2006, which includes macroscopy, cell count, culture, Gram and methylene blue stain, protein and glucose quantitation. CSF culture was performed on 5\% sheep blood agar and chocolate agar. Blood agar was made using Columbia blood agar base (Oxoid, United Kingdom) and 5\% sheep blood, whereas chocolate agar comprised of the same components, with Hemin (factor X) and Nicotinamide adenine dinucleotide (NAD or V factor) released from haemolysed blood [6]. Culture results were read after 24 hours of incubation, and plates without growth were re-incubated for further 24 hours and re-examined for the presence of growth.

Antimicrobial susceptibility testing was done manually from 2010 to 2013 using the Kirby-Bauer disc diffusion method, and on the VITEK ${ }^{\circledR} 2$ (bioMérieux, United States of America) in 2014. Only the antibiotics most commonly used in empirical therapy of meningitis were included in the study, based on the Namibia Standards Treatment Guideline. These were: penicillin, ceftriaxone, chloramphenicol, vancomycin, erythromycin, clindamycin, ciprofloxacin, cotrimoxazole and tetracycline. A bacterial isolate was defined sensitive, intermediate sensitivity or resistant using Clinical Laboratory Standards Institute (CLSI) guidelines on antimicrobial susceptibility [8] [9]. Samples collected in 2014 were analyzed using Real-time PCR and all results including inconclusive results were included in the study for comparison purpose.

\subsection{Methods of Data Analyses}

Data was analyzed using IBM SPSS version 22. Descriptive statistics were used to summarize the frequencies of bacterial organisms isolated from CSF, and their susceptibility patterns to various antimicrobial agents. The frequency of bacterial organisms causing meningitis in children was determined for the period of 2010 to 2014 and displayed in a frequency table. The percentage sensitivity and resistance of bacterial isolates were calculated using all the results from 2010 to 2014 . Inconclusive results, reports with missing data and outliers were excluded from all statistical analysis.

The comparison of culture, PCR, Gram stain, cell count, protein and glucose was performed using chi-square and a $P$ value less than 0.05 was considered significant. The comparison of culture and PCR was done using 2014 data only, while the comparison of culture, Gram stain, cell count, protein and glucose was done on 2010 to 2014 data. All comparison results were displayed in contingency tables.

\section{Ethical Considerations}

Since the study was conducted on de-identified electronic results of CSF tested at NIP, individual patient consent was not required. Authorization to conduct this study was granted by the researches committees of the Ministry of Health and Social Services (MoHSS), NIP and NUST.

\section{Results}

A total of 784 cerebrospinal fluid samples collected from children below five years were examined for suspected bacterial meningitis at NIP from January 2010 to August 2014. Out of the 784 CSF samples, 63 positive cases were detected, which was a positivity rate of $8 \%$. Table 1 displays the individual frequency of each bacterial organism isolated.

S. pneumoniae showed high resistance to penicillin and cotrimoxazole, as well as moderate resistance to erythromycin, clindamycin and tetracycline. All S. pneumoniae isolates were sensitive to ciprofloxacin, vancomycin and ceftriaxone. Table 2 represents the antimicrobial susceptibility patterns of bacteria isolated from CSF of children below 5 years in WHK, 2010-2014.

From the 784 CSF samples, 52 were examined using both culture and PCR, and the results were compared. A positive culture result was defined as isolation of any bacterial organism from CSF and a positive PCR result was defined as detection of any bacterial organism in CSF. Table 3 represents the comparison of culture and 
PCR in the detection of bacterial organisms in CSF.

From the 784 CSF samples of children under 5 years that were examined from 2010 to 2014 at NIP in Windhoek, 762 had both culture and Gram stain results. The results were compared to determine if there is a significant difference between culture and Gram stain. A Gram stain result was considered positive if bacteria were seen on the Gram-stained smear. Table 4 represents the comparison of culture and Gram stain.

Out of a total of 784 CSF samples, 702 had both the neutrophil count and culture results. Table 5 represents the association between the results of culture and the CSF neutrophil count.

Protein estimates and culture results were obtained in 700 of the total 784 CSF samples examined. Of these $700,93.1 \%(\mathrm{n}=652)$ were culture negative while $6.9 \%(\mathrm{n}=48)$ were culture positive. Table 6 represents the association between the results of CSF culture and CSF protein estimates.

Glucose estimates and culture results were obtained in 714 of the total 784 CSF samples examined. Culture was negative in $93.8 \%(n=670)$ and positive in $6.2 \%(n=44)$ cases of the total 714 cases. Table 7 represents the association between the culture results and CSF glucose estimates.

\section{Discussion}

This study aimed at determining the frequency of bacterial organisms isolated from CSF of children below five years and to determine the antimicrobial sensitivity patterns of the four well known leading causes of bacterial meningitis in infants.

The findings of this current study were different from those obtained from a similar study conducted in Mozambique in 2006, whereby $70.6 \%$ of all bacterial organisms isolated from 43 positive cases were $H$. influenzae, S. pneumoniae and N. meningitidis [10]. In the same study, H. influenzae was isolated in $33 \%$ of the positive cases, S. pneumoniae in $21 \%$ cases and $N$. meningitidis in $16 \%$ of the total positive cases. Other causes of bacterial meningitis accounted for $30 \%$ of all positive cases, which included S. aureus $(9.3 \%)$, S. agalactiae (4.65\%), Pseudomonas species (4.6\%), group D streptococcus (4.6\%), E. coli $(2.3 \%)$, Proteus species $(2.3 \%)$ and undetermined Gram negative bacilli (2.3\%) [10]. This is different from our findings where a reduced frequency of bacteria isolated from CSF was reported, as shown in Table 1. This may be due to differences in health care programmes in the two countries.

Table 1. Frequency of bacteria isolated from CSF of children below five years, WHK, 2010 to 2014.

\begin{tabular}{ccc}
\hline Bacterial organisms isolated & Frequency & Percent (\%) \\
\hline S. pneumoniae & 18 & $28.60 \%$ \\
H. influenzae & 4 & $6.30 \%$ \\
N. meningitidis & 2 & $3.10 \%$ \\
S. agalactiae & 1 & $1.60 \%$ \\
K. pneumoniae & 3 & $4.80 \%$ \\
E. coli & 5 & $7.90 \%$ \\
S. aureus & 7 & $11.10 \%$ \\
Pseudomonas species & 1 & $1.60 \%$ \\
Staphylococcus hominis (S. hominis) & 2 & $3.10 \%$ \\
Acinetobactern species & 4 & $6.30 \%$ \\
Serratia species & 3 & $4.80 \%$ \\
Enterococcus species & 3 & $4.80 \%$ \\
Moraxella species & 1 & $1.60 \%$ \\
Enterobacter species & 1 & $1.60 \%$ \\
S. epidermidis & 7 & $11.10 \%$ \\
Tohingomonas paucimobilis (S. paucimobilis) & 1 & $1.60 \%$ \\
Total & 63 & 100 \\
\hline
\end{tabular}


E. L. Haimbodi et al.

Table 2. Antimicrobial susceptibility patterns of bacteria isolated from CSF of children below 5 years in WHK, 2010-2014.

\begin{tabular}{|c|c|c|c|c|c|c|c|c|c|}
\hline Organism isolates & Total isolates & Antibiograms & $\begin{array}{l}\text { Total } \\
\text { tested }\end{array}$ & $\mathrm{S}$ & $\% \mathrm{~S}$ & $\mathrm{R}$ & $\% \mathrm{R}$ & I & $\%$ I \\
\hline \multirow[t]{9}{*}{ S. pneumoniae } & 18 & Penicillin & 17 & 5 & 29.4 & 12 & 70.5 & 0 & 0 \\
\hline & & Ceftriaxone & 8 & 8 & 100 & 0 & 0 & 0 & 0 \\
\hline & & Chloramphenicol & 18 & 17 & 94.4 & 0 & 0 & 1 & 5.5 \\
\hline & & Vancomycin & 13 & 13 & 100 & 0 & 0 & 0 & 0 \\
\hline & & Erythromycin & 18 & 14 & 77.7 & 4 & 22.2 & 0 & 0 \\
\hline & & Clindamycin & 17 & 15 & 88 & 2 & 12 & 0 & 0 \\
\hline & & Ciprofloxacin & 5 & 5 & 100 & 0 & 0 & 0 & 0 \\
\hline & & Cotrimoxazole & 16 & 1 & 6.25 & 15 & 93.7 & 0 & 0 \\
\hline & & Tetracycline & 18 & 14 & 77.7 & 3 & 16.6 & 1 & 6.25 \\
\hline \multirow[t]{3}{*}{ S. agalactiae } & 1 & Penicillin & 1 & 1 & 100 & 0 & 0 & 0 & 0 \\
\hline & & Erythromycin & 1 & 1 & 100 & 0 & 0 & 0 & 0 \\
\hline & & Tetracycline & 1 & 0 & 0 & 1 & 100 & 0 & 0 \\
\hline \multirow[t]{4}{*}{ N. meningitidis } & 2 & Penicillin & 2 & 2 & 100 & 0 & 0 & 0 & 0 \\
\hline & & Ceftriaxone & 2 & 2 & 100 & 0 & 0 & 0 & 0 \\
\hline & & Chloramphenicol & 2 & 2 & 100 & 0 & 0 & 0 & 0 \\
\hline & & Cotrimoxazole & 2 & 0 & 0 & 2 & 100 & 0 & 0 \\
\hline \multirow[t]{5}{*}{ H. influenzae } & 4 & Ceftriaxone & 3 & 3 & 100 & 0 & 0 & 0 & 0 \\
\hline & & Chloramphenicol & 4 & 4 & 100 & 0 & 0 & 0 & 0 \\
\hline & & Cotrimoxazole & 3 & 1 & 33.3 & 2 & 66.6 & 0 & 0 \\
\hline & & Tetracycline & 2 & 1 & 50 & 1 & 50 & 0 & 0 \\
\hline & & Cefuroxime & 2 & 2 & 100 & 0 & 0 & 0 & 0 \\
\hline
\end{tabular}

Abbreviations: $\mathrm{S}=$ sensitive, $\mathrm{R}=$ resistant, $\mathrm{I}=$ intermediate.

Table 3. Comparison of PCR and culture in the detection of bacterial organisms in CSF.

\begin{tabular}{|c|c|c|c|c|}
\hline & & \multicolumn{2}{|c|}{ Culture results } & \multirow{2}{*}{ Total } \\
\hline & & Negative & Positive & \\
\hline \multirow{4}{*}{ PCR results } & Negative & $21(44.7 \%)$ & $1(20 \%)$ & $22(42.3 \%)$ \\
\hline & Positive & $5(10.6 \%)$ & $3(60 \%)$ & $8(15.4 \%)$ \\
\hline & Inconclusive & $21(44.7 \%)$ & $1(20 \%)$ & $22(42.3 \%)$ \\
\hline & Total & $47(100 \%)$ & $5(100 \%)$ & $52(100 \%)$ \\
\hline
\end{tabular}

$\chi^{2}$ value $=8.40(P$ value $=0.015)$

Table 4. Comparison of culture and Gram stain in detection of bacterial organisms in CSF.

\begin{tabular}{|c|c|c|c|c|}
\hline & & \multicolumn{2}{|c|}{ Culture results } & \multirow{2}{*}{ Total } \\
\hline & & Negative & Positive & \\
\hline \multirow{3}{*}{ Gram stain } & Negative & $698(99.3 \%)$ & $19(32.2 \%)$ & $717(94.1 \%)$ \\
\hline & Positive & $5(0.7 \%)$ & $40(67.8 \%)$ & $45(5.9 \%)$ \\
\hline & Total & $703(100 \%)$ & $59(100 \%)$ & $762(100 \%)$ \\
\hline
\end{tabular}

$\chi^{2}$ value $=15.14(P$ value $=0.0001)$. 
Table 5. Association between culture results and the neutrophil count.

\begin{tabular}{ccccc}
\hline & & \multicolumn{2}{c}{ Neutrophil count $\left(\mathrm{mm}^{3}\right)$} & Total \\
\cline { 3 - 4 } & & $<5 \mathrm{~mm}^{3}$ & $>5 \mathrm{~mm}^{3}$ & $665(94.7 \%)$ \\
\multirow{2}{*}{ Culture results } & Negative & $558(98.5 \%)$ & $107(78.8 \%)$ & $37(5.3 \%)$ \\
& Positive & $8(1.4 \%)$ & $29(21.3 \%)$ & $702(100 \%)$ \\
\hline
\end{tabular}

$\chi^{2}$ value $=15.81(P$ value $=0.0001)$

Table 6. Association between CSF culture results and CSF protein.

\begin{tabular}{cccccc}
\hline & & \multicolumn{3}{c}{ CSF protein $(\mathrm{g} / \mathrm{L})$} & Total \\
\cline { 3 - 5 } & & Low & Normal & High & \\
\hline \multirow{2}{*}{ Culture results } & Negative & $381(98.7 \%)$ & $168(95.5 \%)$ & $103(74.6 \%)$ & $652(93.1 \%)$ \\
& Positive & $5(1.30 \%)$ & $8(4.5 \%)$ & $35(25.4 \%)$ & $48(6.9 \%)$ \\
& Total & $386(100 \%)$ & $176(100 \%)$ & $138(100 \%)$ & $700(100 \%)$ \\
\hline
\end{tabular}

$\chi^{2}$ value $=18.42(P$ value $=0.0001)$.

Table 7. Comparison between culture results and CSF glucose level.

\begin{tabular}{|c|c|c|c|c|c|}
\hline & & \multicolumn{3}{|c|}{ CSF glucose $(\mathrm{mmol} / \mathrm{L})$} & \multirow{2}{*}{ Total } \\
\hline & & Low & Normal & High & \\
\hline \multirow{3}{*}{ Culture results } & Negative & $128(81.0 \%)$ & $495(97.6 \%)$ & $47(95.9 \%)$ & $670(93.8 \%)$ \\
\hline & Positive & $30(18.9 \%)$ & $12(2.4 \%)$ & $2(4.1 \%)$ & $44(6.2 \%)$ \\
\hline & Total & $158(100 \%)$ & $507(100 \%)$ & $49(100 \%)$ & $714(100 \%)$ \\
\hline
\end{tabular}

$\chi^{2}$ value $=18.87(P$ value $=0.0001)$.

Studies have shown that group B streptococcus is the leading causative agent of neonatal meningitis, implicated in up to $50 \%$ cases [10]. The findings of this current research however indicated that group B streptococcus was only implicated in $1.6 \%$ of the cases, as indicated in Table 1 . These findings are different from what researchers widely expect and this may be due to frequent antenatal screening in pregnant mothers, to prevent vertical transmission of the organism to the neonates.

S. pneumoniae was the most frequent bacterium isolated from CSF of children below five years in Windhoek. These findings correlate with the findings obtained from a similar study done in Namibia in 2013, whereby $S$. pneumoniae was most frequently isolated from CSF of children aged between 1 - 11 months from 2009-2012 [7]. Similarly, in a study conducted in Mozambique, S. pneumoniae was most frequently isolated from CSF of children under five years [5].

S. pneumoniae showed very high resistance to penicillin and to cotrimoxazole. Furthermore, it exhibited relative resistance to erythromycin and clindamycin ranging from $11.7 \%$ to $22.2 \%$, as shown in Table 2 . These findings correlate with those obtained in a similar study done in Namibia, in which streptococcus were found to be resistant to penicillin and to tetracycline, erythromycin and clindamycin, ranging from $12.4 \%$ to $20.0 \%$ [7].

On the contrary, a similar study conducted in Mozambique found $S$. pneumoniae to be susceptible to penicillin [10]. These findings are different from our study findings, and this may be attributed to differences in pneumococcal strains predominantly causing meningitis in Namibia and Mozambique, which exhibit different sensitivity patterns against penicillin.

S. pneumoniae showed high sensitivity to chloramphenicol, which was also demonstrated in a surveillance study conducted in Mozambique, in which it was susceptible to chloramphenicol [10].

$N$. meningitidis showed high sensitivity to penicillin and chloramphenicol as shown in Table 2, which was different from the findings of a study done in 2013, in which it was found to be $15.2 \%$ resistant to penicillin and $2.9 \%$ resistant to chloramphenicol [7]. However, due to the low number of $N$. meningitidis cases isolated in our 
study, the $100 \%$ sensitivity may be inaccurate; therefore it may portray a wrong picture with regards to susceptibility of this organism to penicillin and chloramphenicol.

H. influenzae showed sensitivity to chloramphenicol and ceftriaxone as well as moderate resistance to tetracycline. This however, differs from findings of a similar study done in Namibia, where $H$. influenzae resistance to chloramphenicol, ceftriaxone and tetracycline was noted [7]. These differences might be attributed to the fact that our study focused on a larger study period of five years while the other study was only for a year.

Group B streptococcus was recorded with the findings of $100 \%$ resistance to tetracycline and $100 \%$ sensitivity to penicillin and erythromycin as shown in Table 2. This is due to a reduced number of Group B streptococcus isolates obtained. An increased number of isolates could portray a different sensitivity pattern.

Although bacterial culture is regarded as a gold standard in the diagnosis of meningitis, the use of antibiotics prior to collection of CSF might attribute to false negative culture results, which therefore necessitates the use of non-culture techniques such as PCR for diagnosis. PCR is the most accurate and reliable method among the non-culture techniques and it is a highly sensitive and specific method which detects specific bacterial DNA in CSF [11].

For the comparison of culture and PCR in this study, the findings of this current study showed that in some culture negative cases, PCR was positive, as shown in Table 3. A similar study was carried out in Syria to compare performance characteristics of PCR and culture in detection of meningitis causing bacteria, in which PCR was positive in $54.5 \%$ of all cases and in $50.9 \%$ of culture negative cases [2]. Our findings were different in this context, and this might be due to differences in culture techniques and PCR methods used.

Studies have shown that Gram stain identifies meningitis causing bacteria in $60 \%-90 \%$ of patients with suspected meningitis [3]. However, in our study, Gram stain was only positive in $5.9 \%$ of the suspected cases as shown in Table 4, which differs significantly from literature. Substandard performance of Gram stain may also be attributed to the use of antibiotics prior to CSF collection. When patients with suspected meningitis are put on antibiotics prior to CSF collection, Gram stain results are likely to be negative. Therefore, Gram stain results were negative in $32.2 \%$ of suspected meningitis cases which were culture positive. Recent treatment with antibiotics can reduce the positivity of Gram stain in culture positive cases, which is observed in Table 4.

Gram stain yields positive results in $10 \%-15 \%$ of patients with bacterial meningitis, who test negative with culture results [11]. The current study found that Gram stain was only positive in $0.7 \%$ of the culture negative cases. However, in a comparative study conducted in Syria, Gram stain was positive by $2.9 \%$ cases with culture negative results [2]. The positivity of Gram stain depends on the concentration of organisms in the CSF, therefore low positivity of Gram stain in true meningitis cases with negative culture results may be obtained.

In this study, Gram stain was positive in $67.8 \%$ of all culture positive cases as shown in Table 4 . Other studies have found that Gram stain was positive in $100 \%$ of all culture positive cases [2]. Decreased Gram stain performance in culture positive cases can be due to an incorrect Gram stain technique applied and reduced number of bacteria present in CSF caused by excessive manipulation of the CSF sample or administration of antibiotics prior to CSF collection [3].

Bacterial meningitis leads to a predominance of neutrophils in CSF in about $80 \%-95 \%$ of bacterial meningitis cases [11]. The findings of this current study however indicated that only $21.3 \%$ of all culture positive cases had a neutrophil count greater than $5 / \mathrm{mm}^{3}$ as shown in Table 5, which is regarded as a significant cut off value in the normal range of CSF neutrophil count [8].

Furthermore, our study found that $1.4 \%$ of samples with bacterial growth had a neutrophil count less than $5 / \mathrm{mm}^{3}$, which does not correlate with indications of bacterial meningitis. Falsely decreased neutrophil count in meningitis can be attributed to the lysis of neutrophils in the CSF sample due to delay in cell count [6].

Our findings indicated that $78.8 \%$ of samples with elevated neutrophil count $\left(>5 / \mathrm{mm}^{3}\right)$ had no bacterial growth. In this case, elevated CSF neutrophil count signifies the presence of bacteria in CSF, however as there was no bacterial growth detected; this might be due to the loss of bacterial viability due to unsuitable transport and storage conditions or excessive manipulation of the CSF sample. Also, WBCs may be present in CSF due to intracranial haemorrhage.

Elevated CSF protein is an indicator of bacterial meningitis. Our study findings showed that only $25.4 \%$ of samples with elevated protein were culture positive, as shown in Table 6, while the rest either had low or normal protein. A greater percentage of CSF samples with elevated CSF protein $(74.6 \%)$ were found to be culture negative, even though elevated CSF protein is suggestive of bacterial meningitis. This may be due to the fact that bacteria lost viability, hence could not be recovered by culture. To the contrary, elevated CSF protein in 
culture negative cases may be a pointer to a different condition such as the flow of plasma protein into the CNS due to intracranial haemorrhage [2].

Laboratory findings of reduced CSF glucose in relation to plasma glucose signify the presence of bacteria in CSF, which metabolises CSF glucose [11]. CSF glucose results alone cannot be used in making decisions pertaining to presence or absence of bacteria in CSF, without comparison with plasma glucose, although it potentially indicates the possibility of bacterial meningitis. Our study therefore only focused on establishing whether or not there is an association between samples with low CSF glucose and culture positive confirmed cases.

We found out that $18.9 \%$ of CSF samples that had low glucose level reported bacterial growth by culture, as shown in Table 7. The low recovery of bacteria in samples with low CSF glucose could be due to antibiotic treatment prior to CSF collection. However, confirmation needed to be made with both CSF and plasma glucose.

\section{Conclusions}

In the current study, we concluded that the most frequent meningitis-causing bacterium isolated from CSF of children below five years in Windhoek was S. pneumoniae. S. pneumoniae was highly resistant to penicillin and cotrimoxazole, and sensitive to ciprofloxacin, vancomycin and ceftriaxone. Although isolated in one case only, group B streptococcus was sensitive to penicillin and resistant to tetracycline.

There was a statistically significant association between CSF culture results and PCR results in detecting meningitis-causing bacteria in CSF. There was also statistically significant association between culture results and Gram stain results.

\section{References}

[1] Best, J. and Hughes, S. (2008) Evidence behind the WHO Guidelines: Hospital Care for Children-What Are the Useful Clinical Features of Bacterial Meningitis Found in Infants and Children? Journal of Tropical Paediatrics, 354, 8386.

[2] Strasinger, S.K. and Di-Lorenzo, M.S. (2008) Urinalysis and Body Fluids. 5th Edition, F. A. Davis Company, Philadelphia.

[3] Fouad, R., Khairy, M., Fathalah, N., Gad, T., El-kholy, B. and Yosry, A. (2014) Role of Clinical Presentations and Routine CSF Analysis in the Rapid Diagnosis of Acute Bacterial Meningitis in Cases of Negative Gram Stained Smears. Journal of Tropical Medicine, 2014, 1-7. http://dx.doi.org/10.1155/2014/213762

[4] Centre for Disease Control and Prevention (2010) Paediatric Bacterial Meningitis Surveillance-African Region 20022008. Morbidity and Mortality Weekly Report, 58, 493-497.

[5] Roca, A., Sigauque, B., Quinto, L., Mandomando, I., Valles, X., Espasa, M. and Alonso, P. (2006) Invasive Pneumococcal Disease in Children Less than Five Years in Rural Mozambique. Tropical Medicine and International Health, 11, 1422-1431. http://dx.doi.org/10.1111/j.1365-3156.2006.01697.x

[6] Winn, W.Jr., Allen, S., Janda, W., Koneman, E., Procop, G., Schreckenberger, P. and Woods, G. (2006) Koneman's Colour Atlas and Textbook of Diagnostic Microbiology. 6th Edition, Lippincott Williams and Wilkins, Philadelphia.

[7] Mengistu, A., Gaeseb, J., Uaaka, G., Ndjavera, C., Kambyambya, K., Indongo, L. and Sangwa, E. (2013) Antimicrobial Sensitivity Patterns of Cerebrospinal Fluid (CSF) Isolates in Namibia: Implications for Empirical Antibiotic Treatment of Meningitis. Journal of Pharmaceutical Policy, 6, 1-10. http://dx.doi.org/10.1186/2052-3211-6-4

[8] Cheesbrough, M. (2006) District Laboratory Practice in Tropical Countries-Part 2. 2nd Edition, Cambridge University Press, New York. http://dx.doi.org/10.1017/CBO9780511543470

[9] Clinical and Laboratory Standards Institute (2014) Performance Standards for Antimicrobial Susceptibility Testing; Twenty-Fourth Informational Supplement. CLSI Document M100-S24. Clinical and Laboratory Standards Institute, Wayne.

[10] Heath, P.T. and Okike, I.O. (2010) Neonatal Bacterial Meningitis: An Update. Paediatrics and Child Health, 20, 526530. http://dx.doi.org/10.1016/j.paed.2010.04.001

[11] Nagarathna, S., Venaakumari, H.B. and Chandramuki, A. (2012) Laboratory Diagnosis of Meningitis. www.intechopen.com http://dx.doi.org/10.5772/29081 


\section{Submit or recommend next manuscript to SCIRP and we will provide best service for you:}

Accepting pre-submission inquiries through Email, Facebook, LinkedIn, Twitter, etc.

A wide selection of journals (inclusive of 9 subjects, more than 200 journals)

Providing 24-hour high-quality service

User-friendly online submission system

Fair and swift peer-review system

Efficient typesetting and proofreading procedure

Display of the result of downloads and visits, as well as the number of cited articles

Maximum dissemination of your research work

Submit your manuscript at: http://papersubmission.scirp.org/ 